\title{
Honeybee Floral Resources in Southwestern Nigeria
}

\author{
Abel Adebayo Ayansola (Corresponding Author) \\ Senior Research Fellow, Entomology Unit, Natural History Museum \\ Obafemi Awolowo University, Ile-Ife, Nigeria
}

Tel: 234-803-352-9966 E-mail: aaayansola@yahoo.com

\author{
Banjo Adedoyin Davies \\ Reader, Department of Plant Science \& Applied Zoology \\ Olabisi Onabanjo University, Ago-Iwoye, Nigeria \\ Tel: 234-803-401-7628Ｅ-mail: adaba55@yahoo.co.uk.
}

Received: April 27, 2012 Accepted: May 11, 2012

doi:10.5296/jbls.v3i1.1720 URL: http://dx.doi.org/10.5296/jbls.v3i1.1720

\begin{abstract}
Every beekeeper is expected to be familiar with the bee floral resources near/ or around his/her apiary for successful beekeeping operation. Understanding the major nectar and pollen producing plants and their flowering period is of great advantage to maximise the efficiency of the bees. Regrettably however, there is dearth of information on floral resources of honeybees in southwestern Nigeria. This study investigated the food-plants of honeybees (Apis mellifera andasonii) in the rainforest and derived savannah zones of southwestern Nigeria between April 2009 and March 2011. Forty nine species of melliferous plants belonging to different families were recorded. The flowering period of each plant species differs from one another. However, there was no month in the year that a plant was not in flower. Differences in bee visitation rates to each plant species were observed. Bee flowering calendar was prepared with the intensity of bee visitation rates. The ethno-medicinal uses of the bee-plants were also documented.
\end{abstract}

Keywords: Melliferous, Honeybee, Floral Resources, Ethno-medicinal, Rainforest, Derived savannah. 


\section{Introduction}

The African honeybee, Apis mellifera andasonii is the major honey producing bee in southwestern Nigeria. It is found in the wild and also, deliberately kept by beekeepers. This bee is an insect that lives in groups or colonies showing social organisations among its castes and in the area of division of labour. It has well developed mouth part and other structural adaptations for collecting nectar and pollen. They generally continue far more than they need and so constantly exude the surplus as honey (Oduntan, 1999). Honeybees visit diverse flowers of plant species, foraging for nectar and pollen. By doing so, bees play a vital role in making plant fertilization possible as well as help in the conservation of biodiversity (Morse and Calderone, 2000). As pollinating agents, bees are environment friendly. Honeybees are useful as samplers of the environment in which they are kept and have been used as bioindicators of ecosystem health (Kevan, 1999). Foraging bees can cover up to eight kilometres radius for pollen, nectar and resinous substances from plants. This activity is an all year round affairs (Aina and Owonibi, 2011). A melliferous plant is a plant which produces substances that can be collected by insects and turned into honey. There are many flowering plants, but not all can be harvested by honey bees, because of their physiognomy (body size and shape, length of proboscis, etc.) In apiculture, a plant is classified as melliferous if it can be harvested by honey bees.

Nectar and pollen are the food of honeybees, which they collect from flowers. Flowering plants are peculiarly noted for the nectaries and nectar secretions. The nectar is most often located within the flower, usually at the base of the sexual column inside the circle of petals. In cotton, however there is a nectariferous ring just outside the base of the petals on the inner base of the calyx. Nectaries are also found outside the flower, on the stem or leaves. Nectar secretion within the flower usually starts about the time the flower opens and ceases soon after fertilisation (Morse and Calderone, 2000).

Beekeepers refer to the heaviest nectar production as honey-flow and most regions have somewhat predictable seasons during which the best nectar-producing flowers are in bloom. These flows can be deceptive, however, since annual fluctuations due to weather conditions or changing crop patterns can result in two or three-fold changes in honey production from one year to the next. Sudden habitat changes can result in dramatic differences in a region's nectar secretion (Oduntan, 1999).

Nectar is a sweet secretion from the nectaries in flowers, and contains 10-60 percent sugar. Using her proboscis, the bee sucks up nectar into her honey stomach, where ferments (enzymes) are added, which invert the sugar to fructose and glucose. The bees carry the nectar to the hive, where they regurgitate it for other bees to transfer into the cells. The bees fan the honey with their wings, and evaporation causes it to become more concentrated (Fenemore and Prakash, 2006).

There is dearth of information on floral resources of honeybees in southwestern Nigeria. However, it is necessary for every beekeeper to be familiar with the melliferous plants near/ or around his/her apiary for successful beekeeping operation. Understanding the major nectar 


\section{Mll Macrothink}

and pollen producing plants and their flowering period is of great advantage to maximise the efficiency of the bees. These explain the rationale for this study.

The objectives of this study were to make an inventory of the bee floral resources in southwestern Nigeria with a view to preparing a checklist of melliferous plants and providing the annual calendar of flowering times of bee-plants. This will assist the beekeepers to properly monitor the nectar flow season within their apiaries. It will also enable a bee farmer to determine when to install bee hives, divide colonies, put on supers, use swarm control measures, remove honey, re-queen, prepare colonies for nectar flow season and locate profitable apiary sites.

\section{Materials and Methods}

A survey of bee flora resources were conducted at both Ilesa (rainforest zone) and Iseyin (derived savannah zone) (Figure 1). Monthly survey of flowering plants were conducted and documented between April 2009 and March 2011. Personal observations were made on bee flora resources during the rain and the dry seasons in the two ecological zones with a view to identifying the major nectar and pollen plants of the study areas. Notes were taken on which plants the bees visit, especially those visited in large numbers. The flowering periods of these plants were recorded. The intensity of visitation of honeybees to flowering plants in the study areas was recorded. Most of the collected plants were identified in the field. Those that could not be identified were identified at the Forestry Research Institute of Nigeria, Ibadan. Information was obtained from beekeepers in the field on the plants providing food for the bees as well as their medicinal uses.

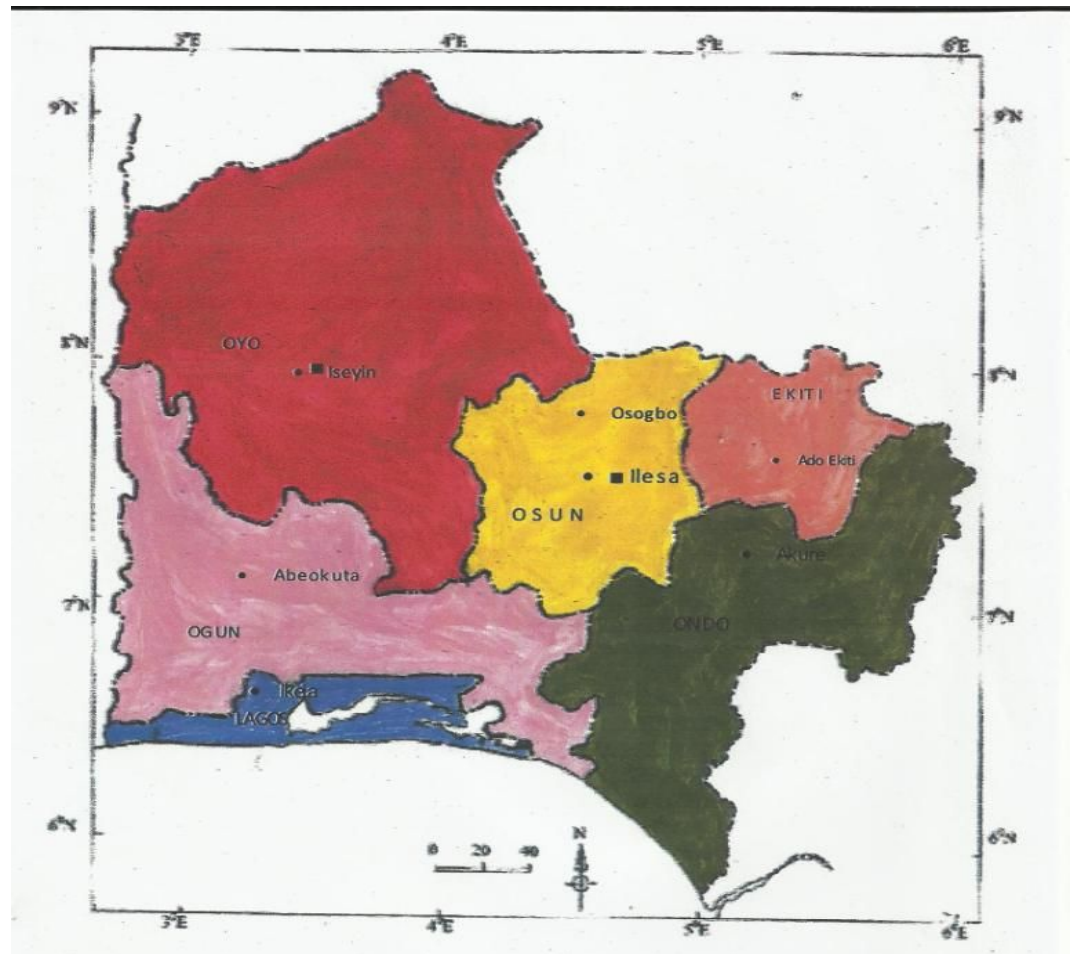

Figure 1. Map of Southwestern Nigeria showing the study areas

Study areas

- State Capitals 


\section{Results}

Bee Flora Resources in the Rainforest and Derived Savannah Ecosystems

The list of flowering plants, flowering times and bee visitation intensity in the rainforest and derived savannah zones of southwestern Nigeria were presented in Tables 1 and 2. Thirty-three species of melliferous plants were recorded and identified in the rainforest zone while 26 species were recorded in the derived savannah zone.

Most of the plant species were cultivated plants (fruits trees, arable crops, and vegetables), weeds, shrubs and timbers. The flowering times of the plants were not all the same. However, there was no month that a plant was not in flower. There were also differences in the bee visitation rates to each plant species. Some plants were occasionally visited; some were often visited while others were abundantly visited (Tables 1 and 2). It is interesting to note that most of the melliferous plants encountered in this study have medicinal values which imply the ethno-medicinal potential of their honey.

In the rainforest zone, the following plants were fully in flowers between April and July (Table 1): Cocos nucifera, Acacia ataxacanthia, Coffea arabica, Abelmoschus esculentus, Hoslundia opposite, Aspilia africana, Capsicum annum, Solanum melongena, Theobroma cacao and Cucurbita maxima. From July to August, Acacia atacanthia, Hoslundia opposite, Aspilia africana, Capsicum annum, Chromolaena odorata, Ananas comosus, Lycopersicum esculentum Manihot esculenta, Musa sapientum, Zea mays, Talinum triangulare, Tridax procumbes, and Treculia africana were abundantly in flower. From October to December, the following plants were fully in flower: Citrus sinesis, Cocos nucifera, Chromolaena odorata, and Garcina cola. From January to February, the following plants were fully in flower: Cocos nucifera, Garcina cola and Tapinanthus sp. (Table 1).

Bee visitation intensity varies from one plant to another (Table 1). Flowers of plants that were abundantly visited $(+++)$ were Cola nitida, Mangifera indica, Coffea arabica, Cola acuminata, Capsicum annum, Alstonia congensis, Cucurbita maxima, Lycopersicon esculentum, Manihot esculenta, Musa sapientum, Struchium sparganophora, Theobroma cacao, Tapinanthus sp., Zea mays and Tridax procumbens. The remaining plants were either often visited $(++)$ or occasionally visited $(+)$ (Table 1$)$.

In the derived savannah zone of southwestern Nigeria (Table 22), the following plants have few flowers from April to June: Amaranthus viridis, Clerodendrum capitalum, Corchorus olitorius, Zea mays and Piper guineese. From July to September, the following plants were fully in flowers: Arachis hypogea, Ananas comosus, Capsicum annum, Carica papaya, Colocasia esculenta and Mahihot esculenta. Between October and December, the flowers of the following plants were in abundance: Allium sativum, Chromolaena odorata, Vernonia amygdalina, and Parkia biglobosa (Table 2). From January to March, Chromolaena odorata and Allium sativum were in flowers. There were differences in the intensity of honeybee visitation rates among different plants (Table 2). Flowers of plants that were abundantly visited $(+++)$ were Allium sativa, Chromolaena odorata, Clerodendrum capitalum, Manihot 
esculenta, Parkia biglobosa, Piper guineese and Zea mays. The other plants were either often visited $(++)$ or occasionally visited.

A checklist of melliferous plants recorded in both rainforest and derived savannah zones of southwestern Nigeria was prepared and presented in Table 3. The species name of each plant, the family name, the local name and medicinal use(s) were recorded. It is noteworthy that the respondents attached ethno-medicinal values to all the melliferous plants.

Table 1. Flowering Times and Bee Visitation Rates in the Rainforest Zone of South Western Nigeria

\begin{tabular}{|c|c|c|c|c|c|}
\hline \multirow{3}{*}{$\begin{array}{l}\text { Plant Species } \\
\\
\text { Citrus sinensis }\end{array}$} & \multicolumn{2}{|c|}{ Rainy Season } & \multicolumn{2}{|c|}{ Dry Season } & \multirow{2}{*}{$\begin{array}{l}\text { Visitation } \\
\text { intensity }\end{array}$} \\
\hline & $\begin{array}{l}\text { April. May } \\
\text { June }\end{array}$ & $\begin{array}{l}\text { July Aug. } \\
\text { Sept }\end{array}$ & $\begin{array}{l}\text { Oct. Nov. } \\
\text { Dec. }\end{array}$ & $\begin{array}{l}\text { Jan. Feb. } \\
\text { March }\end{array}$ & \\
\hline & $\mathrm{V} \quad \mathrm{V}$ & & $\mathrm{V} \quad \mathrm{V} \quad \mathrm{V}$ & $\mathrm{V}$ & $+\quad+$ \\
\hline Cola nitida & $\mathrm{V} \quad \mathrm{V}$ & & $\mathrm{V} \quad \mathrm{V}$ & $\mathrm{V} \quad \mathrm{V}$ & $+\quad+\quad+$ \\
\hline Mangifera indica & & & $\mathrm{V} \quad \mathrm{V}$ & $\mathrm{V} \quad \mathrm{V}$ & $+\quad+\quad+$ \\
\hline Cocos mucifera & $\begin{array}{lll}\mathrm{V} & \mathrm{V} & \mathrm{V}\end{array}$ & $\mathrm{V}$ & $\begin{array}{lll}\mathrm{V} & \mathrm{V} & \mathrm{V}\end{array}$ & $\begin{array}{lll}\mathrm{V} & \mathrm{V} & \mathrm{V}\end{array}$ & $+\quad+$ \\
\hline Acacia ataxacantha & $\begin{array}{lll} & \mathrm{V} & \mathrm{V} \\
\end{array}$ & $\begin{array}{lll}\mathrm{V} & \mathrm{V} & \mathrm{V}\end{array}$ & & $\begin{array}{ll}\mathrm{V} & \mathrm{V}\end{array}$ & \\
\hline Coffea Arabica & $\mathrm{V} \quad \mathrm{V} \quad \mathrm{V}$ & & & & $+\quad+\quad+$ \\
\hline Cola acuminate & $\mathrm{V} \quad \mathrm{V}$ & & & & $+\quad+\quad+$ \\
\hline $\begin{array}{l}\text { Abelmoschus } \\
\text { esculentus }\end{array}$ & $\begin{array}{lll}\mathrm{V} & \mathrm{V} & \mathrm{V}\end{array}$ & $\mathrm{V} \quad \mathrm{V}$ & & & $+\quad+$ \\
\hline Alchornea laxiflora & $\mathrm{V}$ & & & $\mathrm{V} \quad \mathrm{V}$ & + \\
\hline Gossypium barbadense & $\mathrm{V}$ & & $\mathrm{V}$ & $\mathrm{V}$ & + \\
\hline Hoslundia opposite & $\mathrm{V} \quad \mathrm{V} \quad \mathrm{V}$ & $\mathrm{V} \quad \mathrm{V} \quad \mathrm{V}$ & & & $+\quad+$ \\
\hline Aspilia Africana & $\begin{array}{lll}\mathrm{V} & \mathrm{V} & \mathrm{V}\end{array}$ & $\begin{array}{lll}\mathrm{V} & \mathrm{V} & \mathrm{V}\end{array}$ & & & $+\quad+$ \\
\hline Capsicum annum & $\mathrm{V} \quad \mathrm{V} \quad \mathrm{V}$ & $\begin{array}{lll}\mathrm{V} & \mathrm{V} & \mathrm{V} \\
\end{array}$ & $\mathrm{V}$ & & $+\quad+$ \\
\hline Carica papaya & & $\mathrm{V} \quad \mathrm{V}$ & $\mathrm{V}$ & & $+\quad+$ \\
\hline $\begin{array}{l}\text { Chromolaena } \\
\text { modorata }\end{array}$ & & $\mathrm{V} \quad \mathrm{V} \quad \mathrm{V}$ & $\mathrm{V} \quad \mathrm{V} \quad \mathrm{V}$ & & $+\quad+$ \\
\hline Alstonia congensis & $\mathrm{V}$ & & $\mathrm{V}$ & $\mathrm{V} \quad \mathrm{V}$ & $+\quad+\quad+$ \\
\hline Ananas comosus & & $\mathrm{V} \quad \mathrm{V} \quad \mathrm{V}$ & $\mathrm{V}$ & & $+\quad+$ \\
\hline Cucurbita maxima & $\mathrm{V} \quad \mathrm{V} \quad \mathrm{V}$ & & & & +++ \\
\hline Garcinia cola & $\mathrm{V}$ & & $\begin{array}{lll}\mathrm{V} & \mathrm{V} & \mathrm{V} \\
\end{array}$ & $\mathrm{V} \quad \mathrm{V} \quad \mathrm{V}$ & \\
\hline $\begin{array}{l}\text { Lycopersicon } \\
\text { Esculentum }\end{array}$ & $\mathrm{V} \quad \mathrm{V}$ & $\mathrm{V} \quad \mathrm{V} \quad \mathrm{V}$ & $\mathrm{V}$ & & $+\quad+\quad+$ \\
\hline Manihot esculenta & $\mathrm{V}$ & $\begin{array}{lll}\mathrm{V} & \mathrm{V} & \mathrm{V} \\
\end{array}$ & $\mathrm{V}$ & & $+\quad+\quad+$ \\
\hline Musa sapientum & & $\mathrm{V} \quad \mathrm{V} \quad \mathrm{V}$ & $\mathrm{V}$ & & $+\quad+\quad+$ \\
\hline $\begin{array}{l}\text { Struchium } \\
\text { Sparganophora }\end{array}$ & & & $\begin{array}{lll}\mathrm{V} & \mathrm{V} & \mathrm{V}\end{array}$ & $\mathrm{V} \quad \mathrm{V}$ & $+\quad+\quad+$ \\
\hline Terminalia superb & & & $\mathrm{V} \quad \mathrm{V}$ & $\mathrm{V} \quad \mathrm{V}$ & + \\
\hline Solanum melongena & $\begin{array}{lll}\mathrm{V} & \mathrm{V} & \mathrm{V}\end{array}$ & & & & $+\quad+$ \\
\hline
\end{tabular}




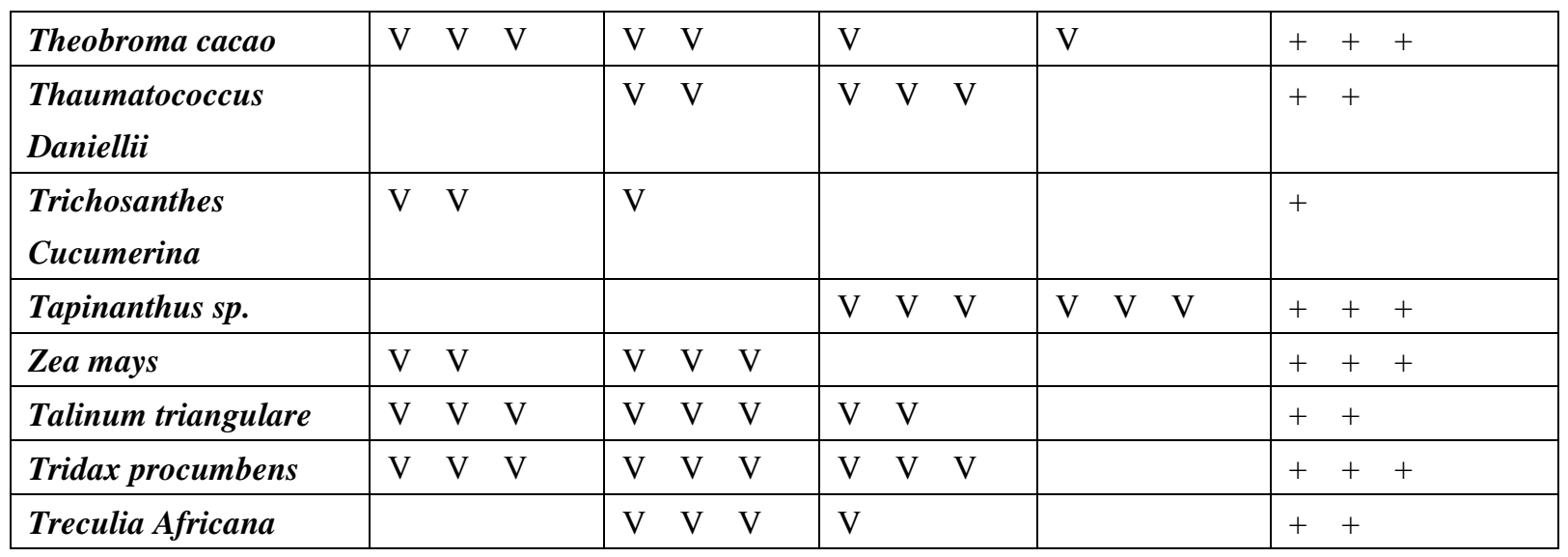

+ Occasionally visited ++ often visited; +++ abundantly visited; $\mathrm{X}$ - flowering time

Table 2. Flowering Times and Bee Visitation Rates in the Derived Savannah Zone of South Western Nigeria

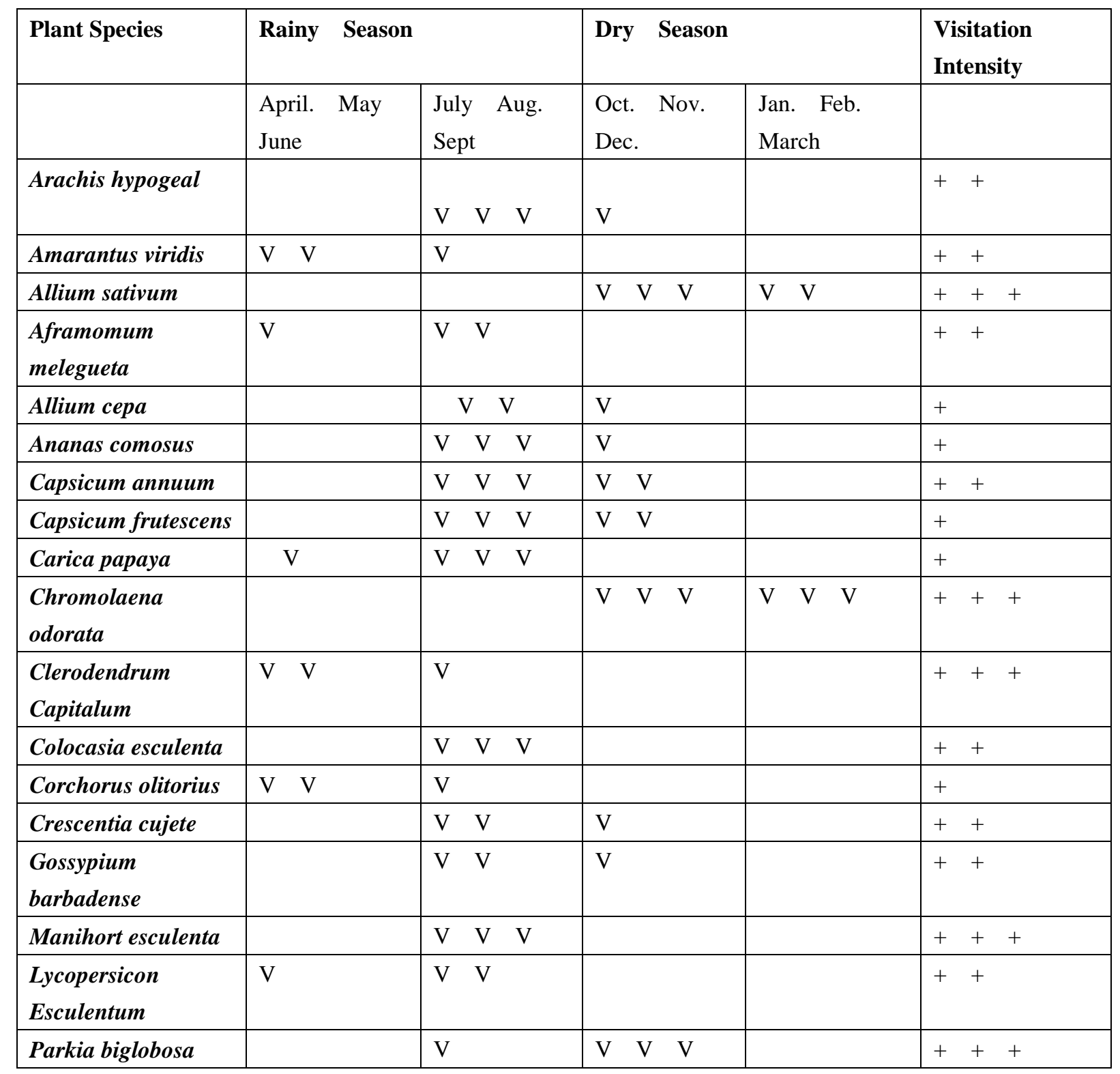




\begin{tabular}{|c|c|c|c|c|}
\hline Phaseolus vulgaris & $\mathrm{V}$ & $\mathrm{V} \quad \mathrm{V}$ & & $+\quad+$ \\
\hline Piper guineese & $\mathrm{V} \quad \mathrm{V}$ & $\mathrm{V}$ & & $+\quad+\quad+$ \\
\hline $\begin{array}{l}\text { Saccharum } \\
\text { Officinarum }\end{array}$ & & $\mathrm{V} \quad \mathrm{V}$ & V V & $+\quad+$ \\
\hline Solanum incanum & $\mathrm{V} \quad \mathrm{V}$ & $\mathrm{V} \quad \mathrm{V} \quad \mathrm{V}$ & & $+\quad+$ \\
\hline Sorghum bicolour & & $\mathrm{V} \quad \mathrm{V}$ & $\mathrm{V}$ & $+\quad+$ \\
\hline $\begin{array}{l}\text { Vernonia } \\
\text { amygdalina }\end{array}$ & & & $\begin{array}{lll}\mathrm{V} & \mathrm{V} & \mathrm{V}\end{array}$ & + \\
\hline Zea mays & V V & V V & & $+\quad+$ \\
\hline
\end{tabular}

+ Occasionally visited; $\quad++$ often visited; $\quad+++$ abundantly visited ; X-flowering time

Table 3. A Checklist of Melliferous Plants recorded in southwestern Nigeria

\begin{tabular}{|c|c|c|c|c|c|}
\hline $\mathbf{S} / \mathbf{N}$ & SPECIES NAME & $\begin{array}{l}\text { FAMILY } \\
\text { NAME }\end{array}$ & $\begin{array}{l}\text { LOCAL } \\
\text { NAME }\end{array}$ & $\begin{array}{l}\text { COMMON } \\
\text { NAME }\end{array}$ & MEDICINAL USE (S) \\
\hline 1 & Citrus sinensis & Rutaceae & $\begin{array}{l}\text { Osanmimu } \\
\text { Oronbo didun }\end{array}$ & Sweet orange & $\begin{array}{l}\text { Dysentery, fever, headache, } \\
\text { antimicrobial agent, anthelmintics, } \\
\text { toothache }\end{array}$ \\
\hline 2 & Cola nitida & Sterculiaceae & $\begin{array}{l}\text { Obi gbanja } \\
\text { Goro }\end{array}$ & Kola & Diarrhoea, stimulant, diuretic \\
\hline 3 & $\begin{array}{l}\text { Mangifera } \\
\text { indica }\end{array}$ & Anacardaceae & Mangoro & Mango tree & $\begin{array}{l}\text { Malaria, high blood pressure, } \\
\text { cough. }\end{array}$ \\
\hline 4 & Cocos nucifera & Palmae & Agbon & Coconut Palm & $\begin{array}{l}\text { Bronchitis, migraine, } \\
\text { dysentery, antiseptic, toothache, } \\
\text { uterine diseases, hair loss, } \\
\text { anthelmintic, laxative, emollient }\end{array}$ \\
\hline 5 & $\begin{array}{l}\text { Acacia } \\
\text { ataxacantha }\end{array}$ & Mimosaceae & $\begin{array}{l}\text { Ihun, } \\
\text { ewon-adele }\end{array}$ & Acacia & Dysentery, backache \\
\hline 6 & Coffea Arabica & Rubiaceae & Kofi & Coffee & Stimulant, rheumatism, diuretic \\
\hline 7 & Cola acuminate & Sterculiaceae & Obi abata & Abata kola & Stimulant, fever, malaria \\
\hline 8 & $\begin{array}{l}\text { Abelmoschus } \\
\text { esculentus }\end{array}$ & Malvaceae & Ila & Okro & $\begin{array}{l}\text { Fevers, dysentery, catarrh } \\
\text { infections, emollient, } \\
\text { antispasmodic, tonic }\end{array}$ \\
\hline 9 & $\begin{array}{l}\text { Alchornea } \\
\text { laxiflora }\end{array}$ & Euuphorbiaceae & Pepe, Ijan & $\begin{array}{l}\text { Three-veined bead } \\
\text { string }\end{array}$ & $\begin{array}{l}\text { Chewing sticks, venereal diseases, } \\
\text { ringworm, antioxidant }\end{array}$ \\
\hline 10 & $\begin{array}{l}\text { Gossypium } \\
\text { barbadense }\end{array}$ & Malvaceae & Owu, & West Indian Cotton & $\begin{array}{l}\text { Convulsions, dysentery, asthma, } \\
\text { hypertension, ulcers, relieve } \\
\text { menstrual cycle disorders, } \\
\text { malaria, ease labour }\end{array}$ \\
\hline 11 & $\begin{array}{l}\text { Hoslundia } \\
\text { opposite }\end{array}$ & Labiatae & Efinrin & Hoslundia & $\begin{array}{l}\text { Conjunctivitis, skin diseases, } \\
\text { jaundice, abdominal pain, } \\
\text { antimicrobial, malaria }\end{array}$ \\
\hline
\end{tabular}




\begin{tabular}{|c|c|c|c|c|c|}
\hline 12 & Aspilia Africana & Compositae & Yunyun & Haemorrhage plant & $\begin{array}{l}\text { Blood clotting, Cleaning sores, } \\
\text { stomach disorders, guinea } \\
\text { worm, gonorrhea, skin rash. }\end{array}$ \\
\hline 13 & Capsicum annum & Solanaceae & $\begin{array}{l}\text { Ata rodo, } \\
\text { sombo, tatase }\end{array}$ & Bell pepper & Carminative, stimulant, Vitamin C \\
\hline 14 & Carica papaya & Caricaceae & Ibepe & Pawpaw & $\begin{array}{l}\text { Gonorrhoea, dysentery, } \\
\text { roundworms, medicinal recipes, } \\
\text { malaria }\end{array}$ \\
\hline 15 & $\begin{array}{l}\text { Chromolaena } \\
\text { odorata }\end{array}$ & Compositae & $\begin{array}{l}\text { Akintola, } \\
\text { Awolowo }\end{array}$ & Siam weed & $\begin{array}{l}\text { Antimicrobial, dysentery, } \\
\text { headache, malaria fever, } \\
\text { toothache, haemostatic, skin } \\
\text { diseases }\end{array}$ \\
\hline 16 & Alstonia congensis & Apocynaceae & Ahun & Stool wool & Malaria, toothache \\
\hline 17 & Ananas comosus & $\begin{array}{l}\text { Bromeliaceae } \\
\text { Araceae }\end{array}$ & Ope-Oyinbo & Pineapple & $\begin{array}{l}\text { Digestion problems, typhoid } \\
\text { fever, cough, anthelmintics }\end{array}$ \\
\hline 18 & Cucurbita maxima & Cucurbitaceae & Elegede & Melon-pumpkin & Tapeworm, diuretic, \\
\hline 19 & Garcinia cola & Guttiferae & Orogbo & Bitter kola & $\begin{array}{l}\text { Antimicrobials, dysentery, } \\
\text { bronchitis, cough, fever, } \\
\text { toothache, throat and respiratory } \\
\text { ailments, stimulant }\end{array}$ \\
\hline 20 & $\begin{array}{l}\text { Lycopersicon } \\
\text { esculentum }\end{array}$ & Solanaceae & Tomati & Tomato & $\begin{array}{l}\text { Earache, carminative, fever, } \\
\text { boils, }\end{array}$ \\
\hline 21 & Manihot esculenta & Euphorbiaceae & Gbaguda, Ege & Cassava & $\begin{array}{l}\text { Gonorrhoea, purgative, } \\
\text { toothache }\end{array}$ \\
\hline 22 & $\begin{array}{c}\text { Musa } \\
\text { sapientum }\end{array}$ & Musaceae & Ogede were & Banana & $\begin{array}{l}\text { Jaundice, typhoid fever, } \\
\text { diarrhea, malaria }\end{array}$ \\
\hline 23 & $\begin{array}{l}\text { Struchium } \\
\text { sparganophora }\end{array}$ & Compositae & Ewuro-odo & Water bitterleaf & headache, malaria \\
\hline 24 & Terminalia superb & Combretaceae & Afara & White afara, & Laxative \\
\hline 25 & $\begin{array}{l}\text { Solanum } \\
\text { melongena }\end{array}$ & Solanaceae & Igba & $\begin{array}{l}\text { Eggfruit, } \\
\text { Long purple }\end{array}$ & Diuretic, purgative, \\
\hline 26 & Theobroma cacao & Sterculiaceae & Koko & Cocoa & Diurectic, blood tonic \\
\hline 27 & $\begin{array}{l}\text { Thaumatococcus } \\
\text { daniellii }\end{array}$ & Marantaceae & Ewe-eeran & Miracle berry & Diabetes, \\
\hline 28 & $\begin{array}{l}\text { Trichosanthes } \\
\text { cucumerina }\end{array}$ & Cucurbitaceae & Tomato-elejo & $\begin{array}{l}\text { English tomato, } \\
\text { Snake gourd }\end{array}$ & Anthelmintic, tonic, fever \\
\hline 29 & Tapinanthus sp. & Loranthaceae & Afomo & Mistletoe & $\begin{array}{l}\text { All purpose herbs, for anaemia, } \\
\text { hypertension, diabetes, stroke, }\end{array}$ \\
\hline 30 & Zea mays & Poaceae & Agbado & Maize & $\begin{array}{l}\text { Gall bladder stone, urinary } \\
\text { troubles, cough, diuretic, } \\
\text { carminative }\end{array}$ \\
\hline
\end{tabular}




\begin{tabular}{|c|c|c|c|c|c|}
\hline 31 & $\begin{array}{l}\text { Talinum } \\
\text { triangulare }\end{array}$ & Portulacaceae & Gbure & Watter Lettuce & $\begin{array}{l}\text { Fresh cuts, high blood pressure, } \\
\text { anaemia }\end{array}$ \\
\hline 32 & $\begin{array}{l}\text { Tridax } \\
\text { procumbens }\end{array}$ & Compositae & $\begin{array}{l}\text { Igbalode, } \\
\text { muwagun, }\end{array}$ & Tridax & $\begin{array}{l}\text { Haemostatic, backache, } \\
\text { stomach-ache }\end{array}$ \\
\hline 33 & Treculia Africana & Moraceae & Afon, Ifoon & African Bread fruit & $\begin{array}{l}\text { Convulsions, ulcers, coughs, } \\
\text { gonorrhea, malaria, anaemia }\end{array}$ \\
\hline 34 & Arachis hypogeal & Leguminosae & Epa & Groundnut, peanut & $\begin{array}{l}\text { Oil as solvent, antimicrobials, } \\
\text { insomnia }\end{array}$ \\
\hline 35 & Amarantus viridis & Amaranthaceae & $\begin{array}{l}\text { Tete- abalaye, } \\
\text { tete-kekere, } \\
\text { atite }\end{array}$ & $\begin{array}{l}\text { Green amaranth, } \\
\text { slender amaranth }\end{array}$ & $\begin{array}{l}\text { Anthelmintics, dysentery, } \\
\text { antispasmodic, diuretic, } \\
\text { gonorrhea, eye disease. }\end{array}$ \\
\hline 36 & Allium sativum & Liliaceae & Ayo, ayuu & Garlic & $\begin{array}{l}\text { Fever, coughs, asthma, } \\
\text { flatulence, anthelmintics, } \\
\text { ringworm, antibiotic, diuretic, } \\
\text { antimicrobials, blood tonic, } \\
\text { malaria }\end{array}$ \\
\hline 37 & $\begin{array}{l}\text { Aframoтит } \\
\text { melegueta }\end{array}$ & Zingiberaceae & Ata-ire, atare & $\begin{array}{l}\text { Alligator pepper, } \\
\text { grains of paradise }\end{array}$ & $\begin{array}{l}\text { Stimulant, smallpox, chicken pox, } \\
\text { anthelmintics, wounds, coughs, } \\
\text { anaemia, rheumatism, measles, } \\
\text { malaria, toothache }\end{array}$ \\
\hline 38 & Allium cepa & Liliaceae & Alubosa & Onion & $\begin{array}{l}\text { Cough, diuretic, anthelmintics, } \\
\text { skin diseases, weak erection, } \\
\text { throat infections }\end{array}$ \\
\hline 39 & $\begin{array}{l}\text { Capsicum } \\
\text { frutescens }\end{array}$ & Solanaceae & $\begin{array}{l}\text { Ata wewe, } \\
\text { ata-eiye }\end{array}$ & $\begin{array}{l}\text { Chili pepper, bird } \\
\text { pepper }\end{array}$ & $\begin{array}{l}\text { Fever, dysentery, stimulant, } \\
\text { carminative, malaria, ingredient of } \\
\text { some herbal recipes }\end{array}$ \\
\hline 40 & $\begin{array}{l}\text { Clerodendrum } \\
\text { capitalum }\end{array}$ & Verbenaceae & Feregede & Hollow stems & $\begin{array}{l}\text { Anthelmintics, dysentery, } \\
\text { gonorrhea }\end{array}$ \\
\hline 41 & $\begin{array}{l}\text { Colocasia } \\
\text { esculenta }\end{array}$ & Araceae & $\begin{array}{l}\text { Koko, kokofun, } \\
\text { koko-ibile }\end{array}$ & $\begin{array}{l}\text { Cocoyam, wild } \\
\text { taro }\end{array}$ & $\begin{array}{l}\text { Anaemia, wounds, rheumatism, } \\
\text { poison antidote }\end{array}$ \\
\hline 42 & $\begin{array}{l}\text { Corchorus } \\
\text { olitorius }\end{array}$ & Tilaceae & Ewedu & Jute plant & $\begin{array}{l}\text { Fever, worms, diarrhea, } \\
\text { anthelmintic, asthma }\end{array}$ \\
\hline 43 & Crescentia cujete & Bignoniaceae & $\begin{array}{l}\text { Igi igba, } \\
\text { igisogba }\end{array}$ & $\begin{array}{l}\text { Calabash tree, } \\
\text { Gourd tree }\end{array}$ & Ingredient for herbal medicines \\
\hline 44 & $\begin{array}{l}\text { Phaseolus } \\
\text { vulgaris }\end{array}$ & Leguminosae & Awuje, Eree & Kidney bean & Antifungal, skin diseases \\
\hline 45 & Piper guineese & Piperaceae & $\begin{array}{l}\text { Iyere, } \\
\text { Ata-iyere }\end{array}$ & $\begin{array}{l}\text { Climbing black } \\
\text { pepper }\end{array}$ & $\begin{array}{l}\text { Herbal recipe ingredient, } \\
\text { rheumatism, stomachache, } \\
\text { anthelmintic, carminative, } \\
\text { impotence, antimicrobials, }\end{array}$ \\
\hline
\end{tabular}




\begin{tabular}{|l|l|l|l|l|l|}
\hline 46 & $\begin{array}{l}\text { Saccharum } \\
\text { officinarum }\end{array}$ & Poaceae & Ireke & Sugar cane & $\begin{array}{l}\text { hypertension } \\
\text { Headache, joint pains, antidote to } \\
\text { snake vom. }\end{array}$ \\
\hline 47 & Solanum incanum & Solanaceae & Ikan, Igba & $\begin{array}{l}\text { Garden egg, egg } \\
\text { plant }\end{array}$ & $\begin{array}{l}\text { gonorrhea, diuretic, purgative, } \\
\text { tonic }\end{array}$ \\
\hline 48 & Sorghum bicolour & Poaceae & Okababa & Millet, guinea corn & Malaria, blood tonic, fever \\
\hline 49 & $\begin{array}{l}\text { Vernonia } \\
\text { amygdalina }\end{array}$ & Compositae & Ewuro & Bitter leaf & $\begin{array}{l}\text { Stomachache, itching, ringworms, } \\
\text { antimicrobial, toothache, } \\
\text { haemostatic, diabetes, pneumonia, } \\
\text { malaria }\end{array}$ \\
\hline
\end{tabular}

\section{Discussion}

Southwestern Nigeria is rich in melliferous plants. In the rainforest zone of Nigeria, many plants were in flower, these include the large forest trees, many ornamental trees, fruit trees and some arable crops (Table 1). Also in flower at this time was the weed, Chromolaena odorata (Syn. Eupatorium odoratum), which was abundant in farm fallows and was much favoured by bees (Table 1).

In the derived savannah zone, honeybees were observed to visit cashew, mango, maize and cassava in abundance (Table 2). During this period, the hum of the bees can be heard all around. Important trees here were Acacia spp. and locust bean (Parkia biglobosa).

The flowering times of the plants were not all the same. However, there was no month that a plant was not in flower. This implies that nectar is available to the bees all the year round and this explains why honey is available throughout the year. However, differences in abundance of flowers at different periods of the year explain the fluctuations that usually occurred in honey flow and availability. This study revealed that honeybees visit different kind of flowers whose plants have their unique medicinal values. This implies that honeys in southwestern Nigeria are multifloral and this explains why honeybee products have diverse nutritional and medicinal properties. It further revealed the reason behind the multipurpose healing power of honey and other bee products.

Pollinators are known to facilitate key ecosystem services such as pollination. Plant-insect interactions such as pollination and seed dispersal benefit the plant populations (Ritchie, 1999), and may greatly influence the plant community structure especially in herbivory (Steffan-Dewenter and Tschamtke, 2002). Furthermore, the vast majority of angiosperms, including agricultural crops are insect pollinated (Kevan, 1999).

Experts have estimated that about $84 \%$ of the crops in the European Union are dependent on insect pollination (Williams, 1996), (Corbet et al., 1991), and almost 25\% of tropical crops depend mostly on bees for pollination (Heard, 1999). Insect groups such as moths, flies, wasps, bees, beetles, butterflies and other invertebrates are critically important for ensuring effective pollination of both cultivated and wild plants (Free, 1993; Roubik, 1995). Most vegetables, nuts and legumes are bee-pollinated; without them many foods would not be available. Examples of such food plants in the tropics include cucumber, coconut, mango, 
pawpaw and oil palm. There are, however, other crops that have self-fertile flowers, which are capable of setting seeds without the help of pollen vectors, but floral visits by pollinators improve both the quality and quantity of their seeds or fruits. Examples are cotton, soya, and tomato (Richards, 2001).

Bees play a major role in maintaining plant biodiversity (Payette, 1996). Majority of plants are dependent on insect pollination for their reproduction. Plants of the same species or varieties but with different genotypes is therefore favoured for crossbreeding. Honey production is thereby quantitatively and qualitatively improved by the genetic exchange. This bee-plant relationship ensures reproduction and plant genetic diversity, while the rewards of insects are nectar and pollen as food from the plants (Gentry, 1982).

One of most well known and studied plant-insect relationship models is between bees and melliferous plants. Due to their harvesting habit, bees are considered to be the most important and efficient plant pollinating insects [Payette, 1996]. Indeed bees harvest ten to 1000 times more pollen than other insects. Individual bees leave the hive 20 to 30 times a day and the hive's bees may visit 20 to 30 million flowers during a season.

In this study, it was observed that there were differences in bee visitation rates to individual flowers. This may be explained by the fact that bees are known to be attracted to flowers that are colourful, contrast well with their background, or have an ultraviolet coloration that serves as a nectar guide (Payette, 1996). This is especially true in the case of red flowers, which bees don't see unless they contain some ultraviolet light pattern, which we don't see. Purple and blue are bees' favourite colours, followed by yellow and orange.

\section{Conclusion}

All the melliferous plants in this study were claimed by the respondents to have one medicinal value or the other. These findings could explain while honeybee products have medicinal properties. In the rainforest and derived savannah zones, there were sufficient melliferous plants to support beekeeping and high yield of honey. There was no month throughout the year that one plant or the other would not be in flower. However, there were differences in bee visitation rates to each of the plants.

A list of major nectar and / or pollen producing plants and their flowering periods in your locality should be a companion. Every beekeeper is expected to be familiar with the vegetation near/ or around his/her apiary for successful beekeeping operation. Understanding the major nectar and pollen producing plants and their flowering period is of great advantage to maximise the efficiency of the bees. It is of great importance for successful beekeeping operation to keep Floral Calendar charts, and plant for bees where necessary. To identify nectar producing plants i.e. honey source, detail information have to be known and quantified, where necessary, the plant medicinal uses, its flowering period and nectar flow, its pollen and its honey production period.

It is important to keep a record of floral calendar for proper monitoring of the nectar flow season within one's own apiary. Useful data on pollen plants have to be studied and a table chart has to be prepared for proper understanding of each plant. The following criteria for the 
selection of bee floral will be found useful: botanical name of plants, family and common names have to be noted. Vegetable form of plants i.e. floral description, distribution and habitat should be of paramount interest, medicinal and other uses as well as nectar rating and its blooming period are also of great importance. Such information also enables a bee farmer to determine when to install packaged bees, divide colonies, put on supers, use swarm control measures, remove honey, re-queen, prepare colonies for nectar flow season and locate profitable apiary sites.

Plan your garden to provide pollen and nectar sources over the entire growing year. Do your best to plant forage for this purpose in relative proximity to your hives. It is best to plant in masses, as single plants may not attract honeybees, which tend to visit only one plant type per foraging trip. Also, single blossoms tend to be easier for honeybees to access than double blossom types.

\section{References}

Aina, D. O., \& Owonibi, K. (2011) Beekeeping Prospects: Palynology and the Environment. Adv. Appl. Sci. Res., 2: 79-85.

Corbet, S. A., Williams I. H., Osborne, J. L. (1991). Bees and the pollination of crops and wild flowers in the European Community. Bee world 72: 47-59.

Fenemore, P.G. \& Prakash, A. (2006). Applied Entomology 2nd Edition. New Age International (P) Limited, Publishers, New Delhi, 270pp.

Free, J. B. (1993). Insect pollination of crops. Academic Press Limited. London.

Gentry, C. (1982). Apiculture de petite e'chelle. Washington DC: Peace Corps, Info. Collect. \& Exch. Man. Se'r. 34

Heard, T. A. (1999). The role of stingless bees in crop pollination. Ann. Rev. Entomol. 44: 183-206.

Kevan, P. G. (1999). Pollinators as bioindicators of state of the environment: species. Activity and diversity. Agric, Ecosys. Envir. 74 pp 373-393.

Morse, R.A. \& Calderone, N.W. (2000). The value of honeybees as pollinators of US. crops in 2000, Pollination Magazine Cornell University, New York

Oduntan, C. (1999). The Practice of Beekeeping. Bee Craft Publishers Abeokuta 110pp.

Payette, A. (1996). Les Apoi“des du Que'bec: Abeilles et agriculture. L'Abeille, 16(4), 14-15.

Richards, A. J. (2001). Does Low Biodiversity Resulting from Modern Agricultural Practice Affect Crop Pollination and Yield? Annals of Botany 88: 165-172

Ritchie, M. E. \& Olff, H. (1999). Herbivory diversity and plant dynamics: compensatory and additive effects. In: Olff, H., Brown, V. K., Drent, R. H. (Eds), Herbivores: Between Plants and Predators. Blackwell Science Ltd, Oxford, pp. 175-204. 


\section{Macrothink}

Journal of Biology and Life Science

ISSN 2157-6076 2012, Vol. 3, No. 1

Roubik, D.W. (1995). Pollination of cultivated plants in the tropics. Agric. serv. Bull., 118. Food and Agriculture Organization Rome. Italy.

Steffan-Dewenter, I. \& Tscharntke, T. (2002). Insect communities and biotic interactions on fragmented calcareous grasslands - a mini review. Biol. Conserv. Vol.104. pp 275-284.

Williams, I. H. (1996). Aspects of bee diversity and crop pollination in the European Union. In: Matheson, A., Buchmann, S. L., O'Toole, C., Westrich, P., Williams, I.H. (Eds), The conservation of Bees. Linnean Soc. Symp. Ser. No. 18, Academic Press, London, pp. 63-80.

\section{Copyright Disclaimer}

Copyright reserved by the author(s).

This article is an open-access article distributed under the terms and conditions of the Creative Commons Attribution license (http://creativecommons.org/licenses/by/3.0/). 